\title{
Composites Containing Ag Nanoparticles for X-ray Protection
}

\author{
Rita PLAIPAITÉ-NALIVAIKO ${ }^{1}$, Diana ADLIENÉ2 ${ }^{2}$, Igoris PROSYČEVAS ${ }^{2,3}$, \\ Valery LUHIN ${ }^{4}$, Tomas GADIŠAUSKAS ${ }^{5}$
}

\author{
${ }^{1}$ Kaunas University of Applied Engineering Sciences, Tvirtoves al. 35, LT - 50155, Kaunas, \\ Lithuania; \\ ${ }^{2}$ Kaunas University of Technology, Studentu str. 50, 51368 Kaunas, Lithuania; \\ ${ }^{3}$ Institute of Materials Science, Kaunas University of Technology, K. Barsausko St. 59, LT-51423 \\ Kaunas, Lithuania; \\ ${ }^{4}$ Belarusian State University of Technology, Sverdlova str. 13a, 220050 Minsk, Belarus; \\ ${ }^{5}$ The General Jonas Žemaitis Miltitary Academy of Lithuania, Silo g. 5A, LT-10322 Vilnius, \\ Lithuania \\ E-mail: ${ }^{1}$ rita.plaipaite@email.com
}

\begin{abstract}
Soft X-rays assisted modification of polymeric nanocomposites containing metal nanoparticles has been performed and radiation induced changes of their physical and chemical properties have been investigated. Thin composite layers were formed on polished glass substrates applying spin-coating technique for composite deposition from solution containing metal powder additives. Synthesis of metal nanoparticles was performed directly in the deposited PMMA layer applying photocatalytic method (UV irradiation), which was followed by sample irradiation with soft X-rays. The surface morphology, composition and microstructure of samples were assessed using scanning electron microscope, energy dispersive X-ray spectroscopy and optical microscopy. Also UV-VIS spectrometry and FTIR spectrometry were applied for characterization of nanocomposites. It was found that the soft X-ray irradiation contributed to the formation of new $\mathrm{Ag}$ particles and their agglomeration into larger clusters within the polymer matrix causing changes of structural and optical properties of polymeric nanocomposites. Mechanisms of low-dose rate X-ray radiation induced structural changes in polymeric composites, processes promoting formation of new particles in polymers; particles growth and clusterization within polymer matrix have been discussed analyzing changes of physical and chemical properties of nanocomposites before and after their X- ray irradiation.
\end{abstract}

KEY WORDS: polymer composites, nanoparticles, poly-methyl-methacrylate (PMMA), soft X-rays

\section{Introduction}

Attention towards nanotechnologies is steadily increasing due to an extensive range of its applications in different fields of science. [1-3]. Metal nanoparticles having size of 1-100 nm play an important role in development of new nanomaterials and composites. The reason of the enforcement for nanoparticles in various applications is their fascinating properties [4-7]. Each metal nanoparticle performs different and has specific properties depending on its size, structure, shape and composition [8]. Nanoparticles aggregate easily because of their high surface energy and are quickly oxidized as well [9]. The optical properties of metal nanoparticles depend on their surface plasmon resonance, where the plasmon refers to the collective oscillation of free electrons within the metal nanoparticle. It is known that the plasmon resonant peak's position and width are sensitive to the size and shape of nanoparticle, the metal species and the surrounding medium $[10,11]$. To beat the aggregation and stability problems, metal nanoparticles are incorporated in a polymer matrix forming nanocomposites.

Polymer nanocomposites are progressive functional materials [12-14] composed of nanoparticles dispersed inside of polymer matrix and covered by polymer, thus forming a skeleton structure. Produced nanocomposites combine the eligible properties of both agents $[1,15,16]$. Silver nanoparticles are the most desirable material used as polymer functionalizing agent, due to their unique properties, that are well established and extensively investigated [2, 17-22]. Nevertheless, practical applications of silver nanoparticles require their entrapment on various substrates and matrixes. In this case polymers are the primarily chosen materials due to their specific morphology, chemical and structural nature with the long polymeric chains allowing incorporation and fine dispersion of nanoparticles. However only suitable functional groups of polymers can be used as targeted reactive sites for the controlled synthesis of nanoparticles [14, 22, 23]. Use of PMMA suggests two close superiorities such as availability to carboxylate functional group for a chemical bonding with the metal ions and high solubility of PMMA in solvent like chloroform for silver chlorate reduction. Actually, there are two synthesis methods of silver-polymer nanostructures: in-situ and ex-situ. The ex-situ method involves silver

Corresponding author.

E-mail address: ${ }^{1}$ rita.plaipaite@email.com 
nanoparticles formation first, followed by dispersion into a polymer matrix. And the in-situ method embraces the metal nanoparticles that can be generated inside a polymer by reduction of metallic precursor which is dissolved in the polymer or the polymerization solution. In-situ technique has been proven to give essential enrichment to the modification of the interfacial area between inorganic particles and the polymer matrix [4, 6, 18, 23-25]. Experience gained investigating silver-polymer nanostructures is of value when dealing with formation and investigation of nanocomposites containing metal particles other than Ag.

Polymer composites are appealing expectants for ionizing radiation armor applications and can be made transparent. In order to capitalize on the superior properties of polymer composites in transparent armor applications [12], novel techniques and fabrication/modification methods are needed. Modification of polymers with high energy radiation (X-rays, gamma rays, electron beam) leads to the formation of new bonds, free radicals, oxidized products, grafts, scission of main chains and cross-linking $[12,14,21,26,27]$. Radiation induced processes have many advantages over other conventional methods. In radiation processing of polymers no catalysts or additives are required to initiate the reaction. Absorption of radiation energy by the backbone polymer initiates generally a free radical process. With chemical initiation free radicals are produced by the decomposition of an initiator into small fragments which attack the base polymer leading to free radicals. On the other hand high dose radiation exposure of polymers leads to the degradation of these structures.

The importance of the research of radiation hardness of polymers is growing up due to the miniaturization of polymeric constructive elements and broader application of polymeric films characterized by modified surface. Functionality and exploitation characteristics of the devices are dependent on the quality and stability of polymeric materials as it is in the case of poly-methyl-methacrylate (PMMA). Radiolysis in the surface layers may significantly change the optical properties of PMMA. The impact of ionizing radiation on polymeric structure (polymer degradation) could be decreased by the formation of polymeric nanocomposites containing metal nanoparticles.

It is well known, that even small irradiation doses may cause alteration of polymer properties at least in the irradiated surface layers when not in the whole volume of the polymer, however only few investigations record the impact of low doses (Gy range) on PMMA properties and there is a lack of information on modification of the structure and properties of low dose irradiated polymer nanocomposites containing metal nanoparticles. Low dose (dose rate) X-ray radiation impact on the optical properties of thin PMMA and nanocomposite Ag/PMMA layers is assessed and discussed in the present paper.

\section{Theoretical Part}

UV irradiation is a widely used technique to produce electrons in the corresponding solution for reduction of metal salts is used for the synthesis of nanoparticles. Within polymer matrix produced electrons reduce metal ions into metal atoms (in present case - Ag+ and Ag0 correspondingly). The produced silver atoms nucleate and grow into silver nanoparticles inside the polymer matrix as it is shown in Figure 1 which was redrawn from [14].

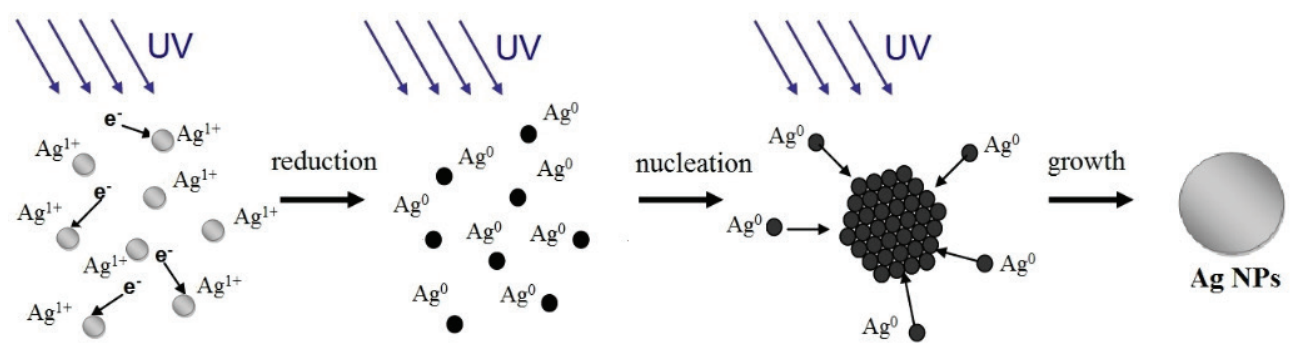

Fig. 1 Schematic representation UV radiation induced reduction of Ag1+ ions to Ag0 atoms and nucleation and growth of Ag nanoparticles in polymer matrix

During the UV irradiation of the polymer, cross-linking process prevails upon polymer scission limiting the growth of the nanoparticles after a critical particle size is reached. The in-situ reduction method doesn't destroy the polymer completely, only a very small part of the polymer is degraded.

The passage of a high energy photon through matter, as it is in the case of its X-ray irradiation, stimulates a complex cascade of processes which results in the dissipation of the primary energy, eventually as thermal energy and chemical reactions. Supposing the high energy photons are the source of radiation, the energy is deposited in a highly abnormal way over a number of processes. If the energy of photons is relatively low $(<35 \mathrm{keV})$ as it was in the case of our investigation, two most important energy losses in polymer mechanisms of photons were represented: Compton scattering and photoelectric effect. In the case of Compton scattering photon interacts with an electron resulting in ejection of the electron and deflection of the photon with reduced energy. The probability of Compton scattering event and the consequent energy of the ejected electron and scattered photon depend on the characteristic energy of the photon and the electron density of the material. In the case of the photoelectric effect, total energy of the photon is absorbed by a single electron. Whereas the energy of the electrons is reduced, there is increased possibility of recombination of cations and secondary electrons to form excited states. The excited-state molecules may return to the ground state through radiation 
less decay, or endure dissociation reactions to form free radicals, which are supposed to be the main agents in further radiochemical reactions. Heterolytic bond decay may in addition result in the formation of charged species or very reactive intermediates. These intermediates can follow several reaction paths, which result in rearrangements and/or formation of new bonds. The ultimate effects of these reactions can be the formation of oxidized products, grafts, scission of main chains (degradation) or cross-linking. The degree of these transformations depends on the structure of the polymer and the conditions of treatment before, during and after irradiation.

The degradation effects of polymers upon exposure to ionizing can be defined as: 1) main-chain scission (degradation) and 2) crosslinking. These are parallel running processes observed in many irradiated polymers, however in certain cases the scission predominates the crosslinking, and such polymers are known as degrading polymers. PMMA belongs to polymer of this type, (Fig. 2.).<smiles>CCC(C)(C)C(=O)OC</smiles><smiles>CCCCCC(C)C</smiles>

Fig.2 Photon beam induced changes in PMMA molecule

The side-chain is initially affected by the photon irradiation and the radical formed is a precursor for the main chain scission. When PMMA is exposed to ionizing radiation, like X-ray irradiation is, a free radical is generated on the ester side-chain, - COOC்H2. A number of different ways are possible for the generation of the side-chain radical:

- by direct action of ionizing radiation: $-\mathrm{COOCH}_{3}+\gamma \rightarrow-\mathrm{COOC}_{2}+\mathrm{H}$;

- by proton transfer of the side-chain cation: $-\mathrm{COOCH}_{3}+\gamma \rightarrow-\mathrm{COOCH}_{3}^{+}+\mathrm{e}^{-}$; and $-\mathrm{COOCH}_{3}^{+} \rightarrow-\mathrm{COOC}_{2}+\mathrm{H}$;

- by hydrogen abstraction: $-\mathrm{COOCH}_{3}+\mathrm{H} \rightarrow-\mathrm{COOCH}_{2}+\mathrm{H}_{2}$.

Radiation induced degradation of PMMA depends on the irradiation dose rate and may lead to the deterioration of mechanical and optical properties.

Two processes affect modification of optical properties upon irradiation of polymer nanocomposites containing metal particles: possible synthesis of new nanoparticles and degradation of polymer structure due to the scission of the main chain.

\section{Instruments and Methods}

Poly-methyl methacrylate (PMMA) solution with metal powder (Ag) additives was prepared dissolving $0.001 \mathrm{M}$ of $\mathrm{AgClO} 4$ in $50 \mathrm{ml}$ of 1\% PMMA solution in chloroform. $\sim 1 \mathrm{~mm}$ thin layers of polymeric structures were spin-coated on the surface of polished optical glass using DYNAPERT PRECIMA centrifuge. Fresh films were dried in the desiccator (air humidity $\sim 30 \%$ ). PMMA films without additives were also produced for the comparison.

Ag/PMMA nanocomposites were produced by in-situ polymerization technique without using any external chemical reagent. PMMA was acting as a protective agent that restricts the mobility of silver ions during the reaction, and hence, agglomeration was mostly controlled. Chloroform was used as a solvent to form chemical network between silver nanoparticles and PMMA. Synthesis of Ag nanoparticles was performed using photocatalytic reduction of silver atoms directly in a thin layer of deposited polymer. UV light source (Hibridas Exposure Unit MA4, power 1200 W, wavelength $300-400 \mathrm{~nm}$ ) was used for this purpose. Applied UV exposure time of $5 \mathrm{~min}$. was sufficient enough for photoreduction of Ag ions and formation of silver nanoparticles. The successful incorporation of Ag nanoparticles was approved and properties of the fabricated layers were investigated performing UV-VIS and FTIR measurements.

UV exposed PMMA and polymeric nanocomposite (Ag/PMMA) films were irradiated to different doses at dose rate of $3.5 \mathrm{mGy} / \mathrm{min}$ using $35 \mathrm{keV}$ X-ray photons generated in X-ray diffractometer DRON-3, which was equipped with a single crystal graphite flat monochromator for transmitting only a narrow $\mathrm{Cu} \mathrm{K} \alpha$ wavelength $(\lambda=0.15405 \mathrm{~nm})$. Irradiation of samples was performed with the aim to investigate soft X-ray radiation induced modification of their optical properties, caused by two processes: polymer degradation and formation of metal nanoparticles within polymer matrix.

Optical properties of experimental films before and after X-ray irradiation were analyzed using Avantes UV/VIS/NIR Avaspec - 2048 spectrometer operating in the wavelength range of $200 \mathrm{~nm}-900 \mathrm{~nm}$ ). Bonding structure of experimental samples was investigated using Fourier Transform infrared spectrometer Bruker Vertex 70, in the wavenumber range from $400 \mathrm{~cm}-1$ to $4000 \mathrm{~cm}-1$, with a resolution of $0.5 \mathrm{~cm}^{-1}$.

The morphology of the samples and bulk composition were investigated in a scanning electron microscope (JSM5610 LV) with attached energy dispersive X-ray analysis (EDX JED-2201; JEOL, Japan) and/or using optical microscope Optika B-600 MET. 


\section{Results and Discussion}

UV-VIS spectroscopy is widely used for the determination of main optical characteristics of different materials that might be evaluated from absorption spectrum taking into account transmittance and reflectance of the light passing through the experimental samples. The absorption spectrum reflects the transitions of electrons from a ground state to an excited state in the molecular orbitals that are appropriate in the wavelength range of investigation. The electronic transitions observed in the UV-VIS spectroscopy generally appear as broad peaks because of the interactions of polymer molecules with each other and the solvent molecules.

The size of synthesized nanoparticles plays also an important role in the setting of the absorption spectrum in UVVIS range, since metal nanoparticles have very specific absorption peaks in the visible region, so called surface plasmon resonance (SPR) band.

UV absorbance spectra of the soft X-ray exposed PMMA layers and PMMA + NPs (Ag) are presented in Fig. 3.

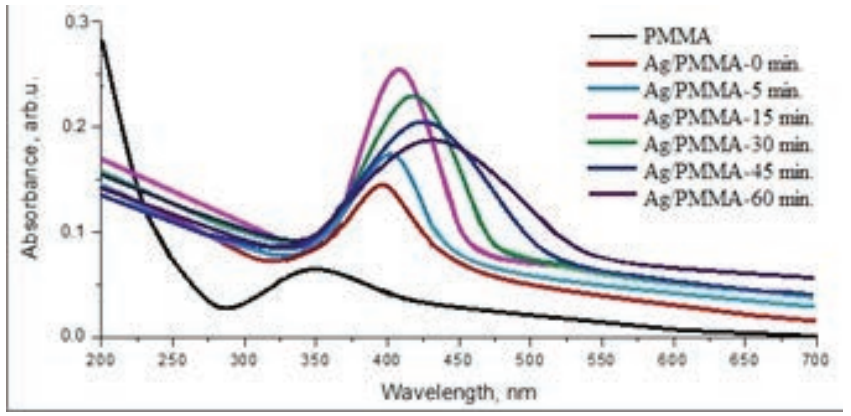

Fig. 3 UV-VIS absorbance spectra of pure PMMA and Ag/PMMA after irradiation with photons at low dose rate

Small peak observed at $436 \mathrm{~nm}$ in absorbance spectrum before X-ray exposure of Ag/PMMA layer indicates that a number of Ag nanoparticles are already present in the polymer composite since they were synthesized during UV preirradiation of samples. Presence of Ag nanoparticles contributes to the increased transparency of nanocomposites as compared to the PMMA. Optical transparency of nanocomposites remains stable in the whole UV-VIS region. Irradiation of experimental samples with soft X-rays shows deterioration of PMMA optical properties due to the degradation of polymer caused by scission of its main chain upon irradiation. Transparency of Ag/PMMA drops down after irradiation with X-rays. The decrease of transparency of X-ray irradiated composites is quantitatively almost the same as for PMMA thus leading to suggestion that radiation induced scission of polymer chain is the main process contributing to deterioration of nanocomposite's optical properties. Nevertheless even after X-ray exposure transparence of Ag/PMMA composites is higher than of PMMA films.

Taking into account that the silver ions can also be reduced by the radicals produced by the degradation of the polymer and that further formation of silver nanoparticles within the polymer matrix is possible, more detailed analysis of absorption/ transmission spectra of irradiated nanocomposites was performed. It was found that irradiation of nanocomposites also leads the redshift of SPR peak from $436 \mathrm{~nm}$ to $451 \mathrm{~nm}$ and broadening of SPR peak. Redshift of SPR peak of metallic particles indicates an increase of metal particle size or formation of the Ag nanoclusters. This is valid also taking into account the electron mean free path effect, according to which the intensity of the SPR band should be higher for bigger particles while the critical size of the surface plasmon mode will be broadened. This is related to the energy transfer from the plasmon to single electron excitation between the quantized levels or to energy dissipation due to inelastic scattering of the transferred electrons, and leads to the strength reduction increasing the size of the metal particles. It should be noted that the exact position and broadening of SPR peak dependent on such parameters as shape of the particle, surface quality, size, and structure.

Investigation of the optical properties of polymer composites has shown that silver nanoparticles were successfully doped in the PMMA matrix. The size synthesized was X-ray dose dependent, however not particles formation but polymer chain scission was the main process contributing to the deterioration of the nanocomposite's optical properties. This suggestion was supported by the results of surface morphology examinations. Surface morphology of experimental polymer films before and after their exposure to soft X-rays is shown in Fig. 4.

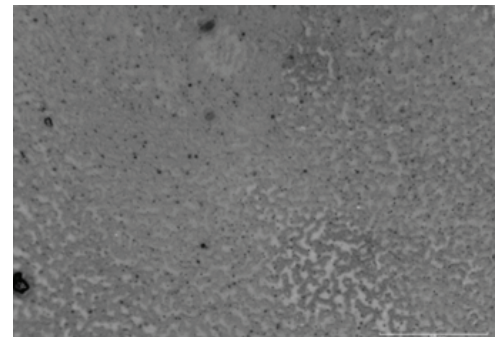

a

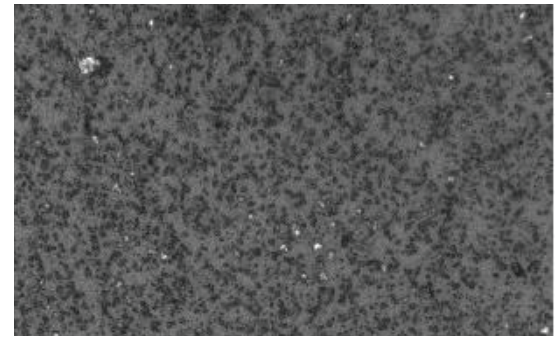

b

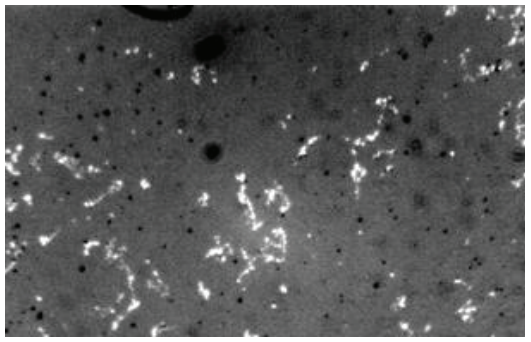

c

Fig.4 Surface morphology of experimental films before their irradiation: a - PMMA, b - Ag/PMMA; and after irradiation to $2 \mathrm{~Gy}: \mathrm{c}-\mathrm{Ag} / \mathrm{PMMA}$ 
It is clearly seen that distributed number of Ag particles are present in the PMMA matrix after its exposure to UV light (Fig. 4b). However X-ray irradiation of the experimental films to doses up to 2 Gy, degradation of PMMA surface and agglomeration of Ag particles to clusters in PMMA matrix (Fig. 4c) was observed. More detailed information on surface morphology was obtained from SEM analysis of the experimental samples. An energy dispersive X-ray analyzer (EDX) was also used in order to get quantitative information about internal content of the samples. SEM images of some irradiated polymer composites that are provided in Fig.5 indicate smooth and well-crystallized deposits of Ag NPs in nanocomposites. In addition, many nodular agglomerated grains (spheres, having diameter of 500-900 nm) that might form or are already forming Ag clusters are clearly seen in nanocomposite layer. It is supposed that a sufficiently uniform distribution of NPs and their agglomeration to some extent may contribute to the increased nanocomposite coatings hardness.
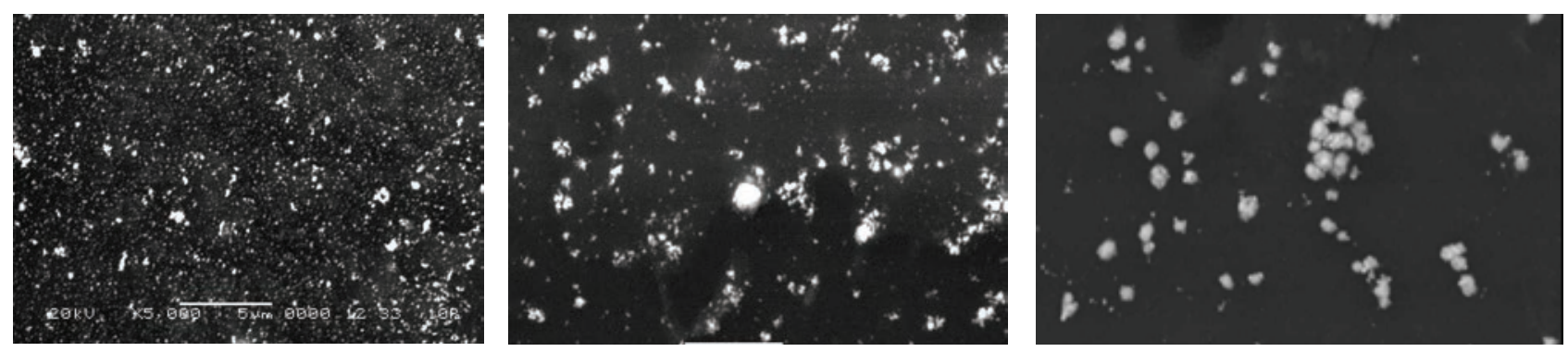

Fig.5 SEM images after films' irradiation to UV light and after film's irradiation to X-rays (0.2 Gy and 2 Gy)

The EDX spectrum and a map of chemical constituents of the nanocomposite coating are provided in Fig. 6 and Fig.7. Clearly expressed Ag peak (69.83\%) indicates formation of metal NPs that agglomerate to clusters.

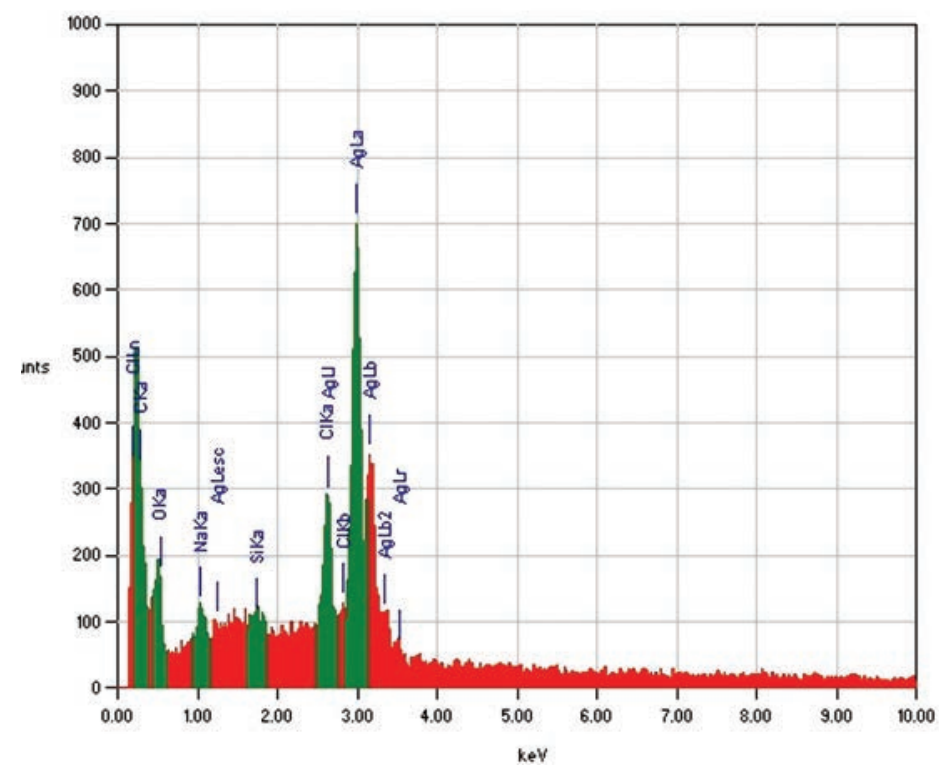

Fig. 6 The EDX spectrum of experimental sample

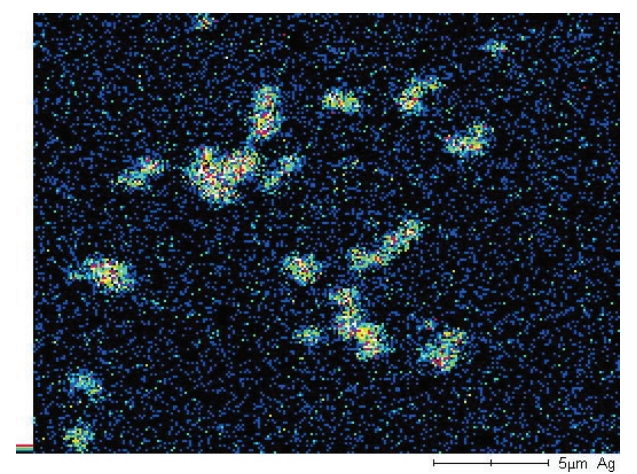

Fig.7 An EDX map of chemical constituents of the nanocomposite coating 
X-ray radiation induced changes in Ag/PMMA composites are linked to the reconfiguration of their bonding structure. Chemical bonding structure of experimental PMMA and nanocomposite (Ag/PMMA) films was investigated before and after their irradiation to the doses up to $2 \mathrm{~Gy}$ by means of infrared spectroscopy, which allows detection of functional groups and characterization of chemical bonds in a molecule [28] by producing an infrared absorption spectrum. FTIR method also provides precise information about orientation of specific functional groups within the polymer film.

The FTIR spectra before and after irradiation of Ag/PMMA films to a certain doses are presented in Fig. 8.

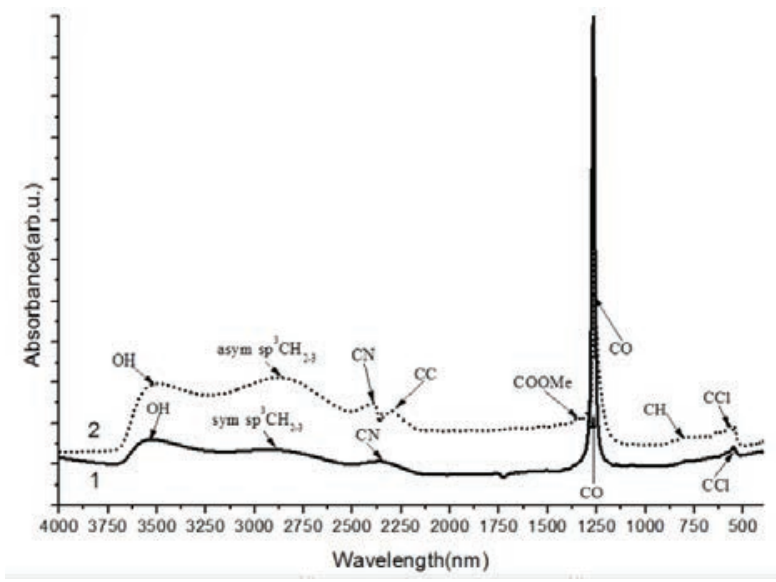

Fig.8 FTIR spectra of Ag/PMMA films before (1) and after (2) their irradiation with X-rays

The FTIR spectra of polymeric nanocomposite (Ag/PMMA) films show sp3CH2-3 (methylene) group in asymmetric stretching and bending mode at $2904 \mathrm{~cm}-1$ which is shifted to lower wavelength at $2865 \mathrm{~cm}-1$ for sp3CH2-3 (methylene) group in symmetric stretching and bending modes after irradiation. It is clearly seen that radiation induces defects and dangling bonds that can be easily occupied by oxygen and create $\mathrm{C}=\mathrm{O}$ and $\mathrm{O}-\mathrm{H}$ bonds. Broad band ranging from 3300 $\mathrm{cm}-1-3700 \mathrm{~cm}-1$ corresponds to the valence and deformation vibrations of $\mathrm{O}-\mathrm{H}$ group. The $\mathrm{C} \equiv \mathrm{N}$ absorption peak observed at $2342 \mathrm{~cm}-1$ split into two peaks 2277 corresponds to the $\mathrm{C} \equiv \mathrm{C}$ group and $2386 \mathrm{~cm}-1$ corresponds to the valence vibrations of nitrile group after irradiation of samples with soft X-rays. It is the evident that the chemical bonds of $\mathrm{N}$ remains in the $\mathrm{Ag} / \mathrm{PMMA}$ nanocomposite structure. The connecting bonding between PMMA and $\mathrm{Ag}$ through $\mathrm{C} \equiv \mathrm{N}$ and $\mathrm{C}=\mathrm{O}$ influences the formation of silver nanoparticles and their size. Thus, PMMA acts as a surfactant and prevents the silver particle aggregation. We assign very intensive absorption peak observed at wave number $1269 \mathrm{~cm}-1$ is due to $\mathrm{C}=\mathrm{O}$ vibration, which after long modification with X-rays decreased and shifted to $1250 \mathrm{~cm}-1$ to $\mathrm{C}-\mathrm{O}$ stretching. The scission process dominates here and due to this the formation of Ag nanoclusters could take place. This occurrence is observed by analyzing OM images after the modification of X-rays.

In the presence of Ag nanoparticles characteristic vibration bands at $\sim 812 \mathrm{~cm}-1$, attributed to out-of-plane vibration of the $\mathrm{C}-\mathrm{H}$ groups. This trend was also observed in the band at $1080 \mathrm{~cm}-1$, assigned to the stretching vibrations of $\mathrm{C}-\mathrm{N}$ group, which indicates that the $\mathrm{N}$ atoms interact with the surface of $\mathrm{Ag}$ nanoparticles by chemical absorption. The fingerprint region shows the $\mathrm{C}-\mathrm{Cl}$ presence in the nanocomposite.

The augmentation in the intensity and appeared peaks of in the FTIR spectra of the investigated samples after 60 min. irradiation indicates that polymer chain reforms itself for the emerging groups and links. This process is part of radiation induced modification of composite structure and properties.

\section{Conclusions}

Series of polymeric (PMMA) and polymeric nanocomposite (Ag/PMMA) samples were investigated and radiation induced changes of their properties were analyzed. It was found that the polymeric nanocomposites containing Ag nanoparticles were more stable upon irradiation, however some degradation of their optical properties was observed.

Results obtained by UV-VIS and FTIR spectroscopies, OM and SEM-EDX analysis indicated that soft X-ray irradiation is suitable for in situ generation of Ag nanoparticles in PMMA matrix; however polymer degradation and metal particle agglomeration are also presented. These processes are related to radiation induced creation of free radicals and unsaturated bonds, which are responsible for possible scission processes in polymers.

FTIR analysis has proven that the interactions in Ag/PMMA films are the result of the coordination bonding between Ag nanoparticles and oxygen.

UV-VIS spectroscopy confirmed the formation of Ag nanoparticles in the polymeric Ag/PMMA nanocomposites due to their X-ray exposure. An interesting reversible peak shift effect of the surface plasmon resonance peak (SPR) was observed in the optical absorbance spectrum when the particle size was changed. It was explained as a versatile competitive result of size effect and extrinsic impact from the matrix or the surrounding medium. The mobility of Ag nanoparticles is augmented and $\mathrm{Ag}$ nanoparticles begin to form $\mathrm{Ag}$ nanoclusters. This is confirmed by the analysis of OM and SEM-EDX images.

Performed investigation shows potential application of Ag NPs containing polymer composites in the implementation 
of surface functionalization strategy: analyzed and discussed soft X-ray induced modification processes in Ag/PMMA composite layers are easily transferable for the analysis of other nanocomposites containing metal nanoparticles. Also assessment of nanocomposite's behavior upon low dose rate irradiation might be of value in applications related to materials that are used in moderate radiation environment.

Acknowledgement: This research is partially funded by a grant (Reg. No. MIP-091/2012 „Optically transparent polymeric nanocomposite shields for radiation protection”) from the Research Council of Lithuania.

\section{References}

1. J.F. Berret, K. Yokota, M. Morvan, R. Schweins, Polymer - Nanoparticle Complexes: From Dilute Solution to Solid State, J Phys Chem B 110 (39) (2006) 19140-19146, doi:10.1021/jp0603177.

2. P. Dallas, V. K. Sharma, R. Zboril, Silver polymeric nanocomposites as advanced antimicrobial agents: Classification, synthetic paths, applications, and perspectives, Adv Colloid Interfac 166 (2011) 119-135, doi:10.1016/j.cis.2011.05.008.

3. S. Prabhu, E.K. Poulose, Silver nanoparticles: mechanism of antimicrobial action, synthesis, medical applications, and toxicity effects, International Nano Letters 2 (2012), doi:10.1186/2228-5326-2-32.

4. Sh. Li, M.M. Lin, M.S. Toprak, D.K. Kim, M. Muhammed, Nanocomposites of polymer and inorganic nanoparticles for optical and magnetic applications, Nano Reviews 1 (2010) 5214, doi:10.3402/nano.v1i0.5214.

5. Ž. Jovanovič, A. Radosavljevič, M. Šiljegovič, N. Bibič, V. Miškovič-Stankovič, Z. Kačarevič-Popovič, Structural and optical characteristics of silver/poly(N-vinyl-2-pyrrolidone) nanosystems synthesized by $\gamma$-irradiation, Radiat Phys Chem 81 (2012) 1720-1728, doi.org/10.1016/j.radphyschem.2012.05.019.

6. D.I. Tee, M. Mariatti, A. Azizan, C.H. See, K.F. Chong, Effect of silane-based coupling agent on the properties of silver nanoparticles filled epoxy composites, Compos. Sci. Technol. 67 (2007) 2584-2591, doi:10.1016/j. compscitech.2006.12.007.

7. V.V. Vodnik, D.K. Božanić, E. Džunuzović, J. Vuković, J.M. Nedeljković, Thermal and optical properties of silver - poly(methylmethacrylate) nanocomposites prepared by in situ radical polymerization, Eur Polym J 46 (2010) 137144, doi:10.1016/j.eurpolymj.2009.10.022.

8. S.T. Knauert, J.F. Douglas, F.W. Starr, The Effect of Nanoparticle Shape on Polymer-Nanocomposite Rheology and Tensile Strength, J Polym Sci B - Polym Phys 45 (14) (2007) 1882-1897, doi:10.1002/polb.21176.

9. A. Tyurin, G. De Filpo, D. Cupelli, F.P. Nicoletta, A. Mashin, G. Chidichimo, Particle size tuning in silverpolyacrylonitrile nanocomposites, Express Polym Lett 4 (2) (2010) 71-78, doi:10.3144/expresspolymlett.2010.12.

10. M. Alsawafta, S. Badilescu, A. Paneri, Vo-Van Truong, M. Packirisamy, Gold-Poly(methyl methacrylate) Nanocomposite Films for Plasmonic Biosensing Applications, Polymers 3 (2011) 1833-1848; doi:10.3390/ polym 3041833.

11. X. Chen, H.J. Schluesener, Nanosilver: A nanoproduct in medical application, Toxicol Lett 176 (2008) 1-12, doi:10.1016/j.toxlet.2007.10.004.

12. D.J.T. Hill, A.K. Whittaker, Radiation chemistry of polymers, Encyclopedia of Polymer Science and Technology 2004, doi:10.1002/0471440264.pst488.

13. V. V. Vodnik, Z. Šaponjič, J.V. Džunuzovič, U. Bogdanovič, M. Mitrič, J. Nedeljkovič, Anisotropic silver nanoparticles as filler for the formation of hybrid nanocomposites, Mater Res Bull 48 (2013) 52-57, doi.org/10.1016/j. materresbull.2012.09.059.

14. E. Yilmaz Preparation and characterization of polymer composites containing gold nanoparticles, PhD thesis, 2011.

15. J. Puišo, D. Adlienė,, A. Guobienė, I. Prosyčevas, R. Plaipaitė-Nalivaiko, Modification of Ag-PVP nanocomposites by gamma irradiation, Mater Sci Eng B 176 (2011) 1562- 1567, doi:10.1016/j.mseb.2011.05.003.

16. J. Oberdisse, Aggregation of colloidal nanoparticles in polymer matrices, Soft Matter 2 (2006) 29-36, doi:10.1039/ B511959F.

17. Y. Liu, Sh. Chen, L. Zhong, G. Wu, Preparation of high-stable silver nanoparticle dispersion by using sodium alginate as a stabilizer under gamma radiation, Radiat Phys Chem 78 (2009) 251-255, doi:10.1016/j.radphyschem.2009.01.003.

18. P.S. Kishore, B. Viswanathan, T.K. Varadarajan, Synthesis and Characterization of Metal Nanoparticle Embedded Conducting Polymer-Polyoxometalate Composites, Nanoscale Res Lett 3 (2008) 14-20, doi:10.1007/s11671-0079107-z.

19. H. Kong, J. Jang, One-step fabrication of silver nanoparticle embedded polymer nanofibers by radical-mediated dispersion polymerization, Chem Commun (2006) 3010-3012, doi:10.1039/b605286j.

20. A.J. Rahedi, J.F. Douglas, F.W. Starr, Model for reversible nanoparticle assembly in a polymer matrix, J Chem Phys 128 (2008) 024902, doi:10.1063/1.2815809.

21. N.H.H. Abu Bakar, J. Ismail, M. Abu Bakar, Silver nanoparticles in polyvinylpyrrolidone grafted natural rubber, React Funct Polym 70 (2010) 168-174, doi:10.1016/j.reactfunctpolym.2009.11.009.

22. M.Z. Kassaee, M.Mohammadkhani, A.Akhavan, R.Mohammadi, In situ formation of silver nanoparticles in PMMA via reduction of silver ions by butylated hydroxytoluene, Struct Chem 22 (2011)11-15, doi:10.1007/s11224010-9671-1. 
23. A. Slistan-Grijalva, R. Herrera-Urbina, J.F. Rivas-Silva, M. Avalos-Borja, F.F. Castillon-Barraza, A. PosadaAmarillas, Synthesis of silver nanoparticles in a polyvinylpyrrolidone (PVP) paste, and their optical properties in a film and in ethylene glycol, Mater Res Bull 43 (2008) 90-96, doi:10.1016/j.materresbull.2007.02.013.

24. R. Udayabhaskar, R.V. Mangalaraja, D. Manikandan, V. Arjunan, B. Karthikeyan, Room temperature synthesis and optical studies on $\mathrm{Ag}$ and $\mathrm{Au}$ mixed nanocomposite polyvinylpyrrolidone polymer films, Spectrochim Acta A 99 (2012) 69-73, doi.org/10.1016/j.saa.2012.08.066.

25. K.M.M. Abou El-Nour, A. Eftaiha, A. Al-Warthan, R.A.A. Ammar, Synthesis and applications of silver nanoparticles, Arabian Journal of Chemistry 3 (2010) 135-140, doi:10.1016/j.arabjc.2010.04.008.

26. N.D. Singho, N.A. Che Lah, M.R. Johan, R. Ahmad, FTIR Studies on Silver-Poly(Methylmethacrylate) Nanocomposites via In-Situ Polymerization Technique, Int J Electrochem Sci 7 (2012) 5596 - 5603.

27. N.D. Singho, N.A. Che Lah, M.R. Johan, R. Ahmad, Enhancement of the Refractive Index of Silver Nanoparticles in Poly (Methyl Methacrylate), International Journal of Research in Engineering and Technology (IJRET) 1 (4) (2012) ISSN 2277 - 4378.

28. J. Coates, Interpretation of infrared spectra, a practical approach, Encyclopedia of analytical chemistry 2005, pp. 10815-10837, doi:10.1002/9780470027318. 